\title{
Nodal and fixed price coexistence in distribution networks \\ with optimal investment planning and tariff design
}

\author{
Iacopo Savelli, Cameron Hepburn \\ Smith School of Enterprise and the Environment, \\ University of Oxford, South Parks Road, Oxford, UK, \\ \{iacopo.savelli, cameron.hepburn\}@ smithschool.ox.ac.uk
}

\author{
Thomas Morstyn \\ School of Engineering, \\ University of Edinburgh, EH9 3FE, Edinburgh, UK, \\ thomas.morstyn@ed.ac.uk
}

\begin{abstract}
The aim of the proposed framework is to show how flexible consumers and small generators paying nodal prices can coexist with traditional consumers paying fixed prices at the distribution grid level. The local grid is managed by a distribution network operator who also determines the lines to be built or expanded. A network tariff levied on grid users is optimally determined to ensure the recovery of both fixed and variable investment network costs. The model is structured as a nonlinear integer bilevel program. The upper level represents a long-term network planning problem accounting for investment costs and network tariffs. The lower level is a market clearing problem, which considers the upper level investment decisions, and determines the cleared quantities and the distribution nodal prices. These values are used in turn by the upper level problem to determine the fixed price paid by traditional consumers and to ensure the recovery of the overall investment costs. The bilevel model is then recast as a mixed-integer quadratically constrained problem by using integer algebra and complementarity relations. The power flows at the distribution grid level are modelled by using a second-order cone relaxation. Numerical tests based on a 18-bus low-voltage distribution network are reported to demonstrate the effectiveness of the proposed approach. In particular, the results show that an increase of demand flexibility can be beneficial also for traditional consumers by triggering a reduction of fixed prices, and can mitigate the subsidising effect between them. Moreover, the optimal network planning shows that a significant welfare increase can be obtained, while ensuring the recovery of both fixed and variable costs through congestion rent and network tariffs levied on grid users.
\end{abstract}

Keywords: distribution nodal prices; network tariffs and charges; fixed cost recovery; flexible and non-flexible consumers.

\section{INTRODUCTION}

Despite the clear benefit in terms of efficiency [1], the deployment of nodal prices at the distribution grid level still faces several issues. Two of the main problems are the volatility and the granularity of nodal prices, i.e. electricity nodal prices can significantly change from one time period to another, and from one location to the other [2]. These issues are exacerbated at the distribution grid level, where distribution nodal prices can differ even within the same local area. For these reasons, traditional consumers can prefer fixed prices [3], as they are unprepared for forecasting volatile prices, or even predicting their own future consumption [4]. However, the adoption of efficient and responsive prices (in particular at the distribution level) is a key requirement for the development of the future "smart" energy system, where flexible consumers and small generators will be able to adjust their consumption and generation as prices change [5].

Electrical networks were historically designed to allow power from large generators connected upstream, to flow downstream through distribution networks to the final consumers [6]. However, the future energy system where consumers and small generators will be actively engaged requires this pattern to be rethought. Indeed, both flexible consumers and prosumers will be able to trade energy and services among themselves, and with the upstream main grid, as in the recently developed energy communities [7], and peer-topeer energy markets [8]. These additional power flows will introduce bidirectional power flows and additional variability on electricity networks, and in particular at the distribution grid level.

Therefore, at least two issues must be addressed to enable a smooth transition to this future energy system. First, nodal prices must be effectively deployed at the distribution grid level, while allowing traditional non-flexible consumers to pay fixed prices. Second, significant network investments will be required to account for the increased power flows. Therefore, new expansion planning strategies must be designed.

In the literature, different aspects of distribution nodal prices have been addressed. A seminal contribution has been proposed by Gan et al. in [9]. They prove that in radial networks a global optimum of the non-linear power flow problem can be obtained by solving a second-order cone program, after slightly reducing the feasible set, and under a mild condition that can be checked a priori, which holds for a wide variety of distribution grids, including the IEEE 13, 34, 37, 123-bus networks. Along this line, reference [10] proposes a hierarchical economic dispatch mechanism involving both national and local markets, where nodal prices at the distribution grid level are modelled by convexifying the AC optimal power flow. A bender-like approach is proposed to coordinate the interactions between layers. Reference [11] 
introduces some explicit sufficient conditions that guarantee the existence and uniqueness of the load-flow solution for distribution networks with a generic topology (radial or meshed). Once the conditions are satisfied, a unique load-flow solution can be reached through a fixed point iteration method. This approach is extended in reference [12] to delta-connected sources/loads, whereas in [13] further linear relations for voltages, line currents, and power injections are provided. This method is used in reference [14] to solve a market clearing problem with a coordinated mechanism for distributed generators and transmission grid interaction. Following from the theory of spot pricing of electricity, popularized by Fred C. Schweppe et al. in [15], reference [16] shows an interesting analysis of distribution nodal prices under a second-order cone relaxation, where the prices are decomposed in terms related to power injections, active and reactive power losses, voltage limits, and transmission constraints.

With respect to network investments, a seminal contribution is due to Paul Joskow and Jean Tirole in [17]. In particular, they focus on several real-world aspects such as the lumpiness of capacity expansions, i.e. network lines can be expanded only by discrete amount. Recently, reference [18] proposes a transmission investment planning problem, where the effect of network tariffs on consumer behaviours is accounted directly into the market clearing problem. Moreover, a fundamental problem in network investment is the recovery of the total investment costs, also termed revenue adequacy condition. In particular, it can be shown that the congestion rent collected by the network operator is not sufficient to ensure the recovery of the fixed investment cost [19]. For this reason, network tariffs must be introduced. An extensive review of the most common tariff schemes is reported in [20] and [21].

The aim of the proposed framework in this paper is to show how traditional non-flexible consumers paying fixed prices can coexist with flexible consumers and small generators subject to nodal prices at the distribution grid level. Fixed prices are determined consistently with the underlying nodal prices to ensure allocative efficiency. In addition, the proposed framework determines how a distribution system operator should optimally manage the local network by determining the lines to be built, and the optimal network tariff levied on grid users. The model is structured as a bilevel program (see e.g. [22]). The upper level is a long-term investment planning problem, which determines the lines to be expanded, while ensuring the recovery of both fixed and variable costs. Given the selected grid expansion, the lower level solves a market clearing problem, which determines the allocated quantities and the distribution nodal prices. These values are used in turn by the upper level to determine the fixed price paid by traditional consumers, and to ensure the revenue adequacy condition. By resorting to standard integer algebra and complementarity relations, the proposed non-linear integer bilevel model is recast as a mixed-integer quadratically constrained problem, which can be solved with off-the-shelf solvers. The power flows at the distribution grid level are modelled by resorting to a second-order cone relaxation, which ensures the exact solution for radial network under mild assumptions [9].

The remaining sections are structured as follows. Section II describes in detail the proposed framework. Section III outlines how the proposed non-linear integer bilevel model can be recast as an equivalent mixed-integer quadratically constrained problem. Section IV reports some test cases obtained by using the proposed model. Finally, Section V summarises the main conclusions.

\section{THE BILEVEL MODEL}

\section{A. Framework description}

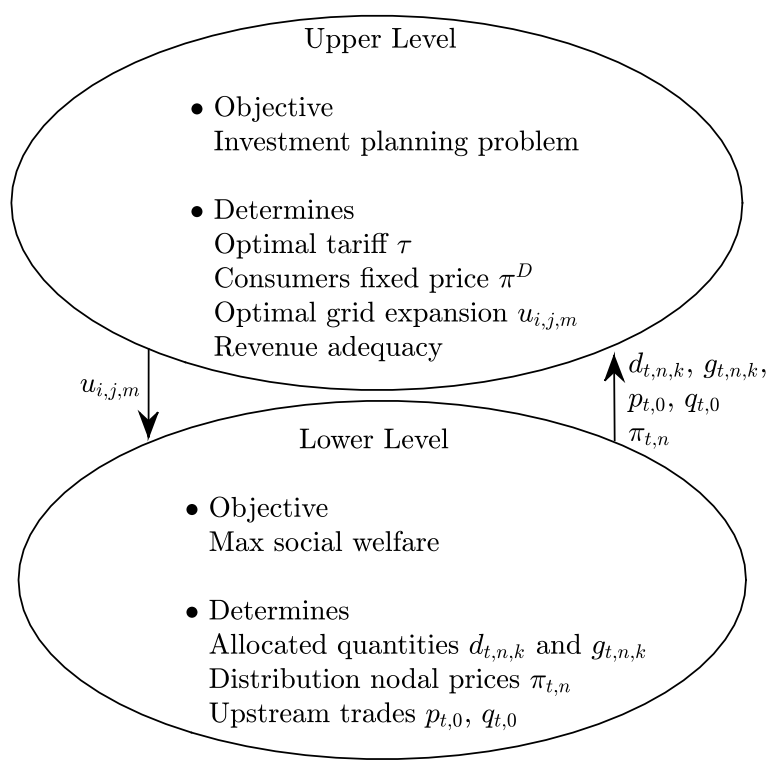

Fig. 1. The proposed bilevel model

The proposed framework is structured as a bilevel model [22]. A bilevel model belongs to the class of hierarchical optimization problems, and it can be regarded as two nested optimization programs, termed upper and lower level problem. Figure 1 outlines the proposed bilevel framework. The upper level is a long-term investment problem. It determines the optimal tariff $\tau$ levied on all network users, and the optimal grid expansion $u_{i, j, m}$. In detail, the terms $u_{i, j, m}$ are binary variables equal to one if the line connecting the node $i$ to the node $j$ is expanded by the lumpy capacity $m \in \mathcal{M}$, and zero otherwise. The finite set of lumpy capacity expansions $\mathcal{M}$ is defined as $\mathcal{M}=\{0 \%, \ldots, 100 \%\}$, where each term represents the percentage of line capacity added with respect to the original one, e.g. $m=100 \%$ means that the line is doubled. The distribution grid is connected upstream with the main transmission network through a substation located at node zero (slack bus). Given the binary variables $u_{i, j, m}$, the lower level program solves a market clearing problem, which determines the trades with the upstream transmission network for both active $p_{t, 0}$ and reactive power $q_{t, 0}$, the allocated local demand $d_{t, n, k}^{p}$ and generation $g_{t, n, k}^{p}$ quantities, and the distribution nodal prices $\pi_{t, n}^{p}$. These values are used in turn by the upper level to compute the fixed price $\pi^{D}$ paid by 
traditional consumers, and to ensure the revenue adequacy condition. The set of all nodes is referred to as $\mathcal{N}$, whereas the term $\mathcal{N}^{+}$is defined as $\mathcal{N} \backslash\{0\}$, i.e. all nodes except the slack bus. The set of lines is termed $\mathcal{L}=\{(i, j), \ldots\}$, where $i$ and $j$ are line node ends. Time periods are indexed by $t \in \mathcal{T}$. Finally, the sets $\Omega_{t, n}^{D}$ and $\Omega_{t, n}^{G}$ with $n \in \mathcal{N}^{+}$, represent the sets of flexible consumers and small generators (paying nodal prices), respectively.

\section{B. Upper level problem}

This section describes in detail the upper level optimization problem. In the following, the starred terms $d_{t, n, k}^{p *}, g_{t, n, k}^{p *}, p_{t, 0}^{*}$, $q_{t, 0}^{*}$, and $\pi_{t, n}^{p *}$ are the optimal decision variables derived from the lower level problem, as outlined in Figure 1. The upper level is a long-term investment planning problem, where the objective function is defined as:

$$
\begin{aligned}
& \max _{\tau, \pi^{D}, u_{i, j, m}} \sum_{t \in \mathcal{T}} \sum_{n \in \mathcal{N}^{+}}\left(\sum_{k \in \Omega_{t, n}^{D}} c_{t, n, k}^{d} d_{t, n, k}^{p *}-\sum_{k \in \Omega_{t, n}^{G}} c_{t, n, k}^{g} g_{t, n, k}^{p *}\right) \\
& -\sum_{t \in \mathcal{T}}\left(c_{t, 0}^{p} p_{t, 0}^{*}+c_{t, 0}^{q} q_{t, 0}^{*}\right) \\
& -\tau \sum_{t \in \mathcal{T}} \sum_{n \in \mathcal{N}^{+}}\left(D_{t, n}+\sum_{k \in \Omega_{t, n}^{D}} d_{t, n, k}^{p, \max }+\sum_{k \in \Omega_{t, n}^{G}} g_{t, n, k}^{p, \max }\right) \\
& -\sum_{(i, j) \in \mathcal{L}} \sum_{m \in \mathcal{M}} u_{i, j, m}\left(K_{i, j, m}^{f i x}+K_{i, j, m}^{v a r} F_{i, j, m}\right) .
\end{aligned}
$$

The upper level decision variables are $u_{i, j, m} \in\{0,1\}$, $\pi^{D} \in \mathbb{R}$, and $\tau \geq 0$. The objective function (1) is composed by four groups of terms enclosed by parentheses. The first one represents the consumers and producers surpluses, where $c_{t, n, k}^{d}$ and $c_{t, n, k}^{g}$ are the submitted bid prices for flexible consumers and generators, respectively. The second group depicts the payments due to the trades with the upstream main grid, for both active $p_{t, 0}$ and reactive $q_{t, 0}$ power, where $c_{t, 0}^{p}$ and $c_{t, 0}^{q}$ are their respective trading prices. The third set of terms refers to the total network tariff levied on all grid users, which is collected by the distribution grid operator. The tariff $\tau$ is paid proportionally to the users' maximum capacity, where $D_{t, n}$ is the fixed demand requested by tradition consumers, whereas $d_{t, n, k}^{p, \max }$ and $g_{t, n, k}^{p, \max }$ are the maximum capacity demanded and supplied by flexible consumers and generators, respectively. The remaining terms represent the overall investment costs, which depend on the selected expansion $u_{i, j, m}$, and include both fixed $K_{i, j, m}^{f i x}$ and variable $K_{i, j, m}^{v a r}$ investment costs, where the latter are paid proportionally to the line capacity built $F_{i, j, m}$.

The recovery of the overall investment costs for the distribution network operator is ensured by enforcing the following revenue adequacy constraint:

$$
\begin{aligned}
& -\sum_{t \in \mathcal{T}}\left(\sum_{n \in \mathcal{N}} \pi_{t, n}^{p *} p_{t, n}^{*}+c_{t, 0}^{q} q_{t, 0}^{*}\right) \\
& +\tau \sum_{t \in \mathcal{T}} \sum_{n \in \mathcal{N}^{+}}\left(D_{t, n}+\sum_{k \in \Omega_{t, n}^{D}} d_{t, n, k}^{p, \max }+\sum_{k \in \Omega_{t, n}^{G}} g_{t, n, k}^{p, \max }\right) \geq
\end{aligned}
$$

$$
K^{o p}+\sum_{(i, j) \in \mathcal{L}} \sum_{m \in \mathcal{M}} u_{i, j, m}\left(K_{i, j, m}^{f i x}+K_{i, j, m}^{v a r} F_{i, j, m}\right),
$$

where the terms in the first line of (2) represent the merchandising surplus, i.e. the difference between the amount paid by consumers and the amount collected by generators [19] (considering also reactive power flows at the slack bus). This difference is a source of revenues for the network operator. The term $K^{o p}$ represents any additional fixed operational cost sustained by the network operator during the considered time period.

To ensure that only one lumpy expansion is selected for each line, the following constraint is added:

$$
\sum_{m \in \mathcal{M}} u_{i, j, m}=1 \quad \forall(i, j) \in \mathcal{L} .
$$

Finally, given the distribution nodal prices obtained by the lover level problem (as sketched in Figure 1), the fixed price $\pi^{D}$ paid by traditional non-flexible consumers is defined as:

$$
\pi^{D} \sum_{t \in \mathcal{T}} \sum_{n \in \mathcal{N}^{+}} D_{t, n}=\sum_{t \in \mathcal{T}} \sum_{n \in \mathcal{N}^{+}} \pi_{t, n}^{p}{ }^{*} D_{t, n} .
$$

That is, the fixed prices $\pi^{D}$ is the average of the nodal prices (for the considered time period and local area), weighted by the fixed demand quantities $D_{t, n}$. Equivalently, the fixed prices $\pi^{D}$ is the value such that the same monetary amount can be collected as if the traditional consumers would pay nodal prices. Therefore, it ensures the payments' balance at the system level. Note that, constraint (4) creates implicitly a subsidy between consumers, where those in lower-priced nodes pay more to compensate those in higher-priced nodes.

\section{Lower level problem}

Given the upper level decision variables on grid expansion $u_{i, j, m}$, the lower level problem actually clears the market at the distribution level by determining the allocated demand $d_{t, n, k}^{p}$ and generation $g_{t, n, k}^{p}$ quantities, and the distribution nodal prices $\pi_{t, n}^{p}$. The objective function of the lover level is defined as:

$$
\begin{aligned}
\max & \sum_{t \in \mathcal{T}} \sum_{n \in \mathcal{N}^{+}}\left(\sum_{k \in \Omega_{t, n}^{D}} c_{t, n, k}^{d} d_{t, n, k}^{p}-\sum_{k \in \Omega_{t, n}^{G}} c_{t, n, k}^{g} g_{t, n, k}^{p}\right) \\
& -\sum_{t \in \mathcal{T}}\left(c_{t, 0}^{p} p_{t, 0}+c_{t, 0}^{q} q_{t, 0}\right)
\end{aligned}
$$

i.e., it represents a social welfare maximization function, considering the trades with the upstream main grid for active $p_{t, 0}$ and reactive $q_{t, 0}$ power requirements.

Active power injections in each node $n \in \mathcal{N}^{+}$with $t \in \mathcal{T}$ are defined as:

$$
p_{t, n}=\sum_{k \in \Omega_{t, n}^{G}} g_{t, n, k}^{p}-\sum_{k \in \Omega_{t, n}^{D}} d_{t, n, k}^{p}-D_{t, n} .
$$

Similar constraints are added for reactive power injections $q_{t, n}$.

To ensure system security, lower and upper voltage magnitude limits $v_{t, n}^{\min }$ and $v_{t, n}^{\max }$ are enforced by using the following constraint:

$$
\left(v_{t, n}^{\min }\right)^{2} \leq W_{t, n} \leq\left(v_{t, n}^{\max }\right)^{2} \quad \forall t \in \mathcal{T}, \forall n \in \mathcal{N}^{+}
$$


where $W_{t, n}$ is the square of the voltage magnitude.

Active power bounds for both flexible consumers and generators are enforced for all $t \in \mathcal{T}$ and $n \in \mathcal{N}^{+}$as:

$$
\begin{array}{ll}
d_{t, n, k}^{p, \min } \leq d_{t, n, k}^{p} \leq d_{t, n, k}^{p, \max } & \forall k \in \Omega_{t, n}^{D} \\
g_{t, n, k}^{p, \min } \leq g_{t, n, k}^{p} \leq g_{t, n, k}^{p, \max } & \forall k \in \Omega_{t, n}^{G},
\end{array}
$$

and similar constraints are added also for reactive power. The power flows at the distribution grid level are modelled by resorting to a well-known second-order cone relaxation, which ensures the exact solution for radial networks under mild assumptions [9]. These constraints are not reported for ease of reading. However, the interested reader is referred to [23] for a detailed example.

\section{MIXED-INTEGER QUADRATICALLY CONSTRAINED EQUIVALENT PROBLEM}

The bilevel model introduced in Section II is composed by two nested optimization programs. However, when the lower level problem is a convex program, it can be equivalently represented by using its first-order necessary and sufficient Karush-Kuhn-Tucker (KKT) conditions [24]. Furthermore, as long as the Slater's condition holds [25], the KKT complementarity conditions (which are non-linear relations) can be equivalently replaced by the strong duality [26], which is a linear relation. The lower level program described in Section II-C is a second-order cone problem (SOCP), i.e., a convex program. Furthermore, for well-formed real-world SOCPs the Slater's condition can be assumed to hold (see [27, p. 89] for a discussion on this point). As a consequence, the described reformulation can be applied, and the proposed lower level problem can be equivalently represented by using (i) the strong duality property, (ii) its primal constraints, and (iii) its dual constraints, as depicted in Figure 2. By inserting

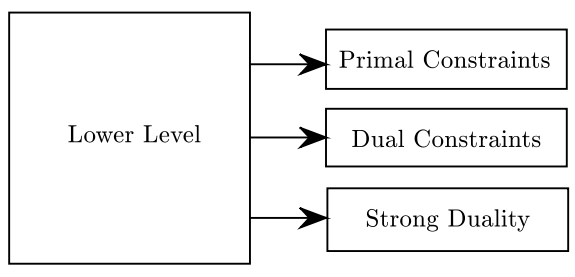

Fig. 2. Lower-level reformulation method

these conditions directly into the upper level program, a single optimization problem equivalent to the proposed bilevel model can be obtained. Finally, by using standard integer algebra [28], and complementarity relations [23], the single level model can be further recast as a mixed-integer quadratically constrained problem (MIQCP), which can be solved with offthe-shelf solvers.

\section{Numerical Results}

This section reports some test cases obtained by applying the MIQCP described in Section III to the 18-bus low-voltage network depicted in Figure 3 [29]. This network is connected upstream with the main grid through a substation, which is

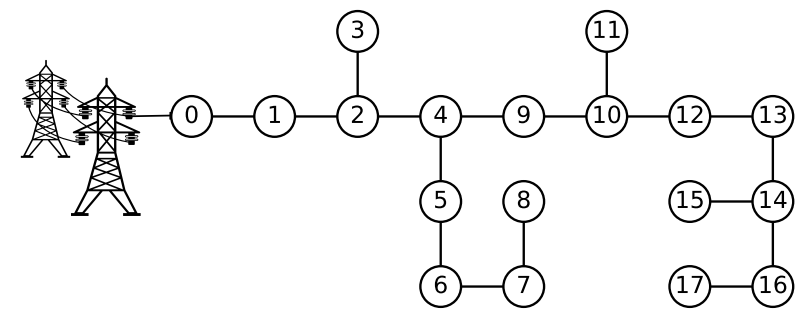

Fig. 3. 18-bus low voltage network [29]

located at node zero (slack bus). Maximum and minimum voltage magnitude in p.u. are $v_{t, n}^{\max }=1.20$ and $v_{t, n}^{\min }=0.80$, respectively. For ease of reading, a single time period is considered, i.e. $\mathcal{T}=\{1\}$. Flexible consumers' bid prices $c_{t, n, k}^{d}$ are sampled from a normal distribution with mean $50 £ / \mathrm{kWh}$ and standard deviation $10 \mathrm{f} / \mathrm{kWh}$. Maximum active power flow over each line is $410 \mathrm{~kW}$. The MIQCP model has been implemented in Python by using Pyomo 5.6 [30], and solved with Cplex 12.9 [31] on a 64-core CPU with 64 GB of RAM.

\section{A. Effect of different proportion between traditional and flex- ible consumers}

The purpose of this test case is to assess the impact on welfare, prices, and executed quantities of different proportions between non-flexible and flexible consumers. The former demand fixed quantities $D_{t, n}$ and pay a fixed price $\pi^{D}$, whereas the latter pay nodal prices $\pi_{t, n}^{p}$ with cleared quantities $d_{t, n, k}^{p}$. In this test case, line expansions are not considered, i.e. $\mathcal{M}=\{0\}$. Consumers (both flexible and non-flexible) are located in nodes $n \in\{3,8,11,15,17\}$. Two different scenarios are compared. In the first, traditional consumers demand fixed quantities $D_{t, n}=60 \mathrm{~kW}$, whereas flexible consumers demand up to $d_{t, n, k}^{p, \max }=20 \mathrm{~kW}$. These quantities are switched in the second scenario. That is, traditional consumers demand $D_{t, n}=20 \mathrm{~kW}$, whereas flexible consumers demand up to $d_{t, n, k}^{p, \max }=60 \mathrm{~kW}$.

TABLE I

EFFECT OF DIFFERENT PROPORTION FLEXIBLE/NON-FLEXIBLE CONSUMERS ON PRICES AND CLEARED QUANTITIES

\begin{tabular}{|c|c|c|c|c|c|c|c|}
\hline \multirow{2}{*}{$n$} & \multirow{2}{*}{$c_{t, n, k}^{d}$} & \multicolumn{3}{|c|}{ Scenario 1 } & \multicolumn{3}{c|}{ Scenario 2 } \\
\cline { 3 - 8 } & & $D_{t, n}=60, d_{t, n, k}^{p, \max }=20$ & \multicolumn{2}{c|}{$D_{t, n}=20, d_{t, n, k}^{p, \max }=60$} \\
\cline { 3 - 8 } & $D_{t, n}$ & $\pi_{t, n}^{p}$ & $d_{t, n, k}^{p}$ & $D_{t, n}$ & $\pi_{t, n}^{p}$ & $d_{t, n, k}^{p}$ \\
\hline 3 & 44.72 & 60 & 44.72 & 17 & 20 & 42.24 & 60 \\
\hline 8 & 42.39 & 60 & 44.86 & 0 & 20 & 42.39 & 47 \\
\hline 11 & 64.62 & 60 & 47.39 & 20 & 20 & 43.95 & 60 \\
\hline 15 & 61.34 & 60 & 61.34 & 16 & 20 & 55.29 & 60 \\
\hline 17 & 48.28 & 60 & 53.41 & 0 & 20 & 48.28 & 27 \\
\hline
\end{tabular}

Table I reports both the nodal prices $\pi_{t, n}^{p}$, and the allocated quantities $d_{t, n, k}^{p}$ in the two scenarios. As can be observed, the increase of flexible demand in the second scenario significantly reduces all nodal prices $\pi_{t, n}^{p}$. As consequence, the fixed price $\pi^{D}$ decreases from $50.34 £ / \mathrm{kWh}$ to $46.43 £ / \mathrm{kWh}$. That is, the increase in flexible consumption is also beneficial for 
traditional non-flexible consumers. In both scenarios, the price in node 15 is the highest, as the line $(14,15)$ has a significantly greater resistance than the other lines. Furthermore, the flexible consumers in nodes 8 th and 17 th become the marginal users in the second scenario [19], their bids $c_{t, n, k}^{d}$ set the nodal prices, and their cleared quantities $d_{t, n, k}^{p}$ rise from zero to $47 \mathrm{~kW}$ and $27 \mathrm{~kW}$, respectively.

Figure 4 shows the difference between the fixed price $\pi^{D}$ paid by traditional consumers and the actual nodal price $\pi_{t, n}^{p}$ in the consumer's location. Recall that the fixed price $\pi^{D}$ is defined by constraint (4) as:

$$
\pi^{D} \sum_{t \in \mathcal{T}} \sum_{n \in \mathcal{N}^{+}} D_{t, n}=\sum_{t \in \mathcal{T}} \sum_{n \in \mathcal{N}^{+}} \pi_{t, n}^{p} D_{t, n}
$$

i.e. a weighted average of the nodal prices during the time period and locations considered. Therefore, traditional nonflexible consumers located in nodes where the nodal price is smaller than the fixed price will pay more by preferring a fixed price. This will implicitly generate a subsidy between non-flexible consumers, where those in lower-priced nodes will pay more to compensate those in higher-prices nodes (see Figure 4 and Figure 6), where the overall monetary equilibrium is ensured by constraint (4). As can be observed by comparing the two scenarios in Figure 4, the increase in flexible consumption reduces this subsidising effect.

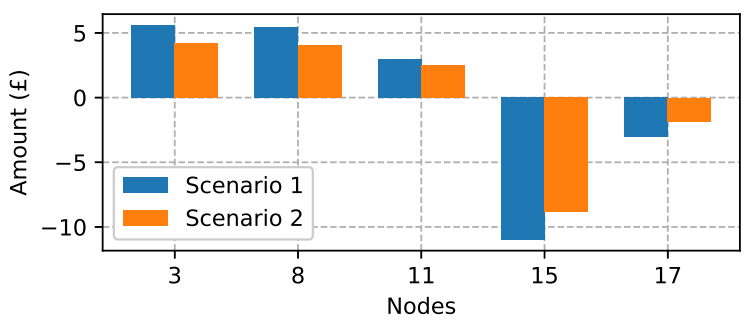

Fig. 4. Difference between the fixed price $\pi^{D}$ paid by traditional consumers and the actual nodal price $\pi_{t, n}^{p}$ in the consumer's location. In the first scenario, $D_{t, n}=60 \mathrm{~kW}$ and $d_{t, n, k}^{p, \max }=20 \mathrm{~kW}$, whereas the fixed price $\pi^{D}$ is $50.34 \mathrm{f} / \mathrm{kWh}$. In the second scenario, $D_{t, n}=20 \mathrm{~kW}$ and $d_{t, n, k}^{p, \max }=60 \mathrm{~kW}$, with $\pi^{D}=46.43 \mathfrak{f} / \mathrm{kWh}$. Nodal prices are depicted in Figure 6 .

\section{B. Effect of distribution line expansions}

In both previous scenarios, the network line between node zero and node one is congested. Therefore, in this third scenario we consider the same settings as in the second one, but we allow all lines to be expanded by a discrete amount (i.e. lumpy expansions), with $\mathcal{M}=\{0 \%, 100 \%\}$. That is, a line can be either left as it is, or doubled. The cost parameters are $K_{i, j, m}^{f i x}=100, K_{i, j, m}^{v a r}=0.1$, and $K^{o p}=0$. In this case, the obtained optimal investment solution suggests to fully expand the lines $(0,1),(1,2),(2,4),(4,9)$, and $(14,15)$, depicted as thick red lines in Figure 5. This leads to a welfare increase from $831.36 £$ to $1,351.32 £$, despite the total investment costs paid. In this case, no line is congested and the fixed price $\pi^{D}$ drops from $46.43 \mathrm{f} / \mathrm{kWh}$ to $40.15 £ / \mathrm{kWh}$, due to the significant nodal price decrease, as shown in Figure 6.

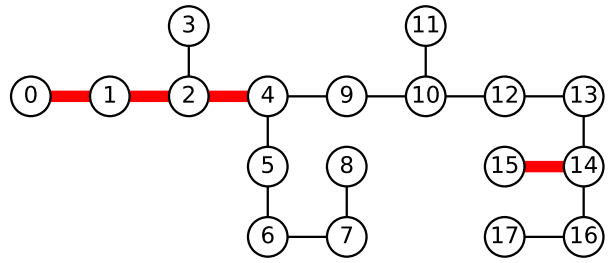

Fig. 5. The optimal expansion plan shows hat the lines marked in red, i.e. the lines $(0,1),(1,2),(2,4),(4,9),(14,15)$, must be fully expanded.

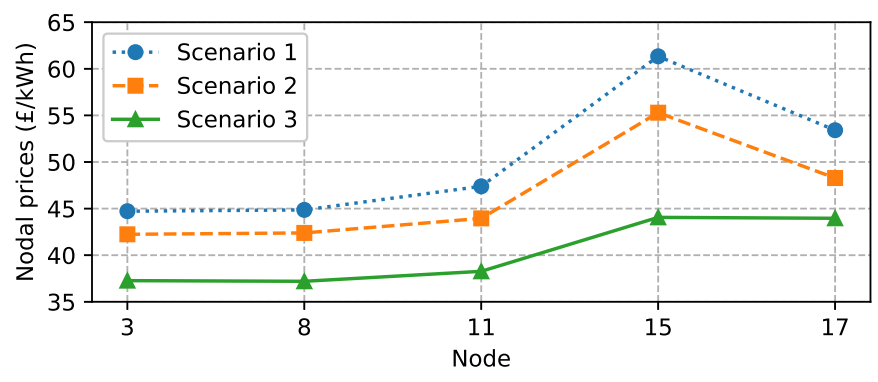

Fig. 6. Comparison between nodal prices in each scenario. In the first scenario, $D_{t, n}=60 \mathrm{~kW}$ and $d_{t, n, k}^{p, \max }=20 \mathrm{~kW}$. In the second scenario, $D_{t, n}=20 \mathrm{~kW}$ and $d_{t, n, k}^{p, \max }=60 \mathrm{~kW}$. The third scenario has the same settings as scenario 2 , but all lines can be expanded with $\mathcal{M}=\{0 \%, 100 \%\}$.

The revenues collected in the considered period $(2,295 £)$ due to the congestion rent are greater than the overall investment costs $(564 £)$, and therefore no network tariff is charged, i.e. $\tau=0 £ / \mathrm{kWh}$. However, if the operational cost $K^{o p}$ rises from zero to 2,000 , then a tariff $\tau=0.59 £ / \mathrm{kWh}$, must be levied on grid users to ensure the revenue adequacy condition (2).

\section{CONCLUSION}

The proposed framework shows how traditional consumers paying fixed prices can coexist with users paying nodal prices at the distribution grid level. Furthermore, it outlines how a local network operator can optimally manage a distribution grid by selecting the lines to be expanded, and the network tariff levied on grid users to ensure the recovery of both fixed and variable investment costs. The test cases analysed show how the increase of flexible demand can lead to a decrease in nodal prices, which can be also beneficial for traditional consumers due to (i) the reduction of their fixed price paid, and (ii) the mitigation of the subsidising effect between them. Finally, the optimal network planning shows that despite the investment costs, a significant welfare increase can be obtained by allowing all lines to be expanded, while ensuring the recovery of both fixed and variable costs through congestion rent and network tariffs.

Further work will aim to extend the proposed framework to include storage devices, as well as to model interactions with upstream wholesale and ancillary service markets.

\section{ACKNOWLEDGEMENT}

The present work has been supported by the EPSRC grant EP/S000887/1 and by the EPSRC grant EP/S031901/1. Icons are from Freepik by Flaticon. 


\section{REFERENCES}

[1] R. Green, "Nodal pricing of electricity: how much does it cost to get it wrong?" Journal of Regulatory Economics, vol. 31, no. 2, pp. 125-149, 2007.

[2] M. Sahni, R. Jones, and Y. Cheng, "Beyond the crystal ball: locational marginal price forecasting and predictive operations in us power markets," IEEE Power and Energy Magazine, vol. 10, no. 4, pp. 35-42, 2012.

[3] D. Biggar and A. Reeves, "Network pricing for the prosumer future: Demand-based tariffs or locational marginal pricing?" in Future of Utilities Utilities of the Future. Elsevier, 2016, pp. 247-265.

[4] M. Fotouhi Ghazvini, J. Soares, H. Morais, R. Castro, and Z. Vale, "Dynamic pricing for demand response considering market price uncertainty," Energies, vol. 10, no. 9, p. 1245, 2017.

[5] D. R. Biggar and M. R. Hesamzadeh, The economics of electricity markets. John Wiley \& Sons, Chichester, UK, 2014.

[6] A. J. Wood, B. F. Wollenberg, and G. B. Sheblé, Power generation, operation, and control. John Wiley \& Sons, 2013.

[7] B. Cornélusse, I. Savelli, S. Paoletti, A. Giannitrapani, and A. Vicino, "A community microgrid architecture with an internal local market," Applied Energy, vol. 242, pp. 547-560, 2019.

[8] T. Morstyn, N. Farrell, S. J. Darby, and M. D. McCulloch, "Using peer-to-peer energy-trading platforms to incentivize prosumers to form federated power plants," Nature Energy, vol. 3, no. 2, pp. 94-101, 2018.

[9] L. Gan, N. Li, U. Topcu, and S. H. Low, "Exact convex relaxation of optimal power flow in radial networks," IEEE Transactions on Automatic Control, vol. 60, no. 1, pp. 72-87, 2014.

[10] Z. Yuan, M. R. Hesamzadeh, and D. R. Biggar, "Distribution locational marginal pricing by convexified acopf and hierarchical dispatch," IEEE Transactions on Smart Grid, vol. 9, no. 4, pp. 3133-3142, July 2018.

[11] C. Wang, A. Bernstein, J. Le Boudec, and M. Paolone, "Existence and uniqueness of load-flow solutions in three-phase distribution networks," IEEE Transactions on Power Systems, vol. 32, no. 4, pp. 3319-3320, July 2017.

[12] A. Bernstein, C. Wang, E. Dall' Anese, J. Le Boudec, and C. Zhao, "Load flow in multiphase distribution networks: Existence, uniqueness, nonsingularity and linear models," IEEE Transactions on Power Systems, vol. 33, no. 6, pp. 5832-5843, Nov 2018.

[13] A. Bernstein and E. Dall'Anese, "Linear power-flow models in multiphase distribution networks," in 2017 IEEE PES Innovative Smart Grid Technologies Conference Europe (ISGT-Europe), Sep. 2017, pp. 1-6.

[14] S. Hanif, K. Zhang, and T. Hamacher, "Coordinated market mechanism for economic dispatch in active distribution grids," in 2019 IEEE Power \& Energy Society General Meeting (PESGM). IEEE, 2019.

[15] F. C. Schweppe, M. C. Caraminis, R. O. Tabors, and R. Bohn, Spot pricing of electricity. Kluwer Academic Publishers, Norwell, MA, 1988.

[25] S. Boyd and L. Vandenberghe, Convex optimization. Cambridge University Press, New York, 2004.
[16] A. Papavasiliou, "Analysis of distribution locational marginal prices," IEEE Transactions on Smart Grid, vol. 9, no. 5, pp. 4872-4882, 2017.

[17] P. Joskow and J. Tirole, "Merchant transmission investment," The Journal of industrial economics, vol. 53, no. 2, pp. 233-264, 2005.

[18] I. Savelli, A. De Paola, and F. Li, "Ex-ante dynamic network tariffs for transmission cost recovery," Applied Energy, vol. 258, p. 113979, 2020.

[19] D. S. Kirschen and G. Strbac, Fundamentals of power system economics. John Wiley \& Sons, 2004.

[20] J. M. Lima, "Allocation of transmission fixed charges: an overview," IEEE Transactions on Power Systems, vol. 11, no. 3, pp. 1409-1418, 1996.

[21] D. Shirmohammadi, B. Gorenstin, M. V. Pereira et al., "Some fundamental, technical concepts about cost based transmission pricing," IEEE Transactions on Power Systems, vol. 11, no. 2, pp. 1002-1008, 1996.

[22] J. F. Bard, Practical bilevel optimization: Algorithms and applications, ser. Nonconvex Optimization and Its Applications. Springer, Boston, MA, 1998, vol. 30.

[23] I. Savelli and T. Morstyn, "Electricity prices and tariffs to keep everyone happy: a framework for compatible fixed and nodal structures to increase efficiency," arXiv preprint arXiv:2001.04283, 2020.

[24] S. A. Gabriel, A. J. Conejo, J. D. Fuller, B. F. Hobbs, and C. Ruiz, Complementarity modeling in energy markets, ser. Int. Series in Operations Research \& Management Science. Springer-Verlag, New York, 2013, vol. 180.

[26] M. S. Lobo, L. Vandenberghe, S. Boyd, and H. Lebret, "Applications of second-order cone programming," Linear algebra and its applications, vol. 284, no. 1-3, pp. 193-228, 1998.

[27] MOSEK ApS, MOSEK Modeling Cookbook, Sep. 2019.

[28] I. Savelli, B. Cornélusse, A. Giannitrapani, S. Paoletti, and A. Vicino, "A new approach to electricity market clearing with uniform purchase price and curtailable block orders," Applied Energy, vol. 226, pp. 618-630, 2018.

[29] S. Papathanassiou, N. Hatziargyriou, K. Strunz et al., "A benchmark low voltage microgrid network," in Proceedings of the CIGRE symposium: power systems with dispersed generation. CIGRE, 2005, pp. 1-8.

[30] W. E. Hart, C. D. Laird, J.-P. Watson, D. L. Woodruff, G. A. Hackebeil, B. L. Nicholson, and J. D. Siirola, Pyomo - Optimization modeling in Python, 2nd ed., ser. Springer Optimization and Its Applications. Springer Science \& Business Media, New York, 2017, vol. 67.

[31] IBM-ILOG, CPLEX User's Manual - Version 12 Release 7, 2016.

[32] I. Savelli, A. Giannitrapani, S. Paoletti, and A. Vicino, "An optimization model for the electricity market clearing problem with uniform purchase price and zonal selling prices," IEEE Transactions on Power Systems, vol. 33, no. 3, pp. 2864-2873, 2018. 